\title{
Finite element analysis of mechanics of neovessels with intraplaque hemorrhage in carotid atherosclerosis
}

\author{
Jinqiu Lu', Wanying Duan², Aike Qiao ${ }^{1 *}$
}

\footnotetext{
* Correspondence: qak@bjut.edu.cn ${ }^{1}$ College of Life Science and Bioengineering, Beijing University of Technology, Beijing, 100124 China
}

\begin{abstract}
Background: Intraplaque hemorrhage is a widely known factor facilitating plaque instability. Neovascularization of plaque can be regarded as a compensatory response to the blood supply in the deep intimal and medial areas of the artery. Due to the physiological function, the deformation of carotid atherosclerotic plaque would happen under the action of blood pressure and blood flow. Neovessels are subject to mechanical loading and likely undergo deformation. The rupture of neovessels may deteriorate the instability of plaque. This study focuses on the local mechanical environments around neovessels and investigates the relationship between the biomechanics and the morphological specificity of neovessels.
\end{abstract}

Methods: Stress and stretch were used to evaluate the rupture risk of the neovessels in plaque. Computational structural analysis was performed based on two human carotid plaque slice samples. Two-dimensional models containing neovessels and other components were built according to the plaque slice samples. Each component was assumed to be non-linear isotropic, piecewise homogeneous and incompressible. Different mechanical boundary conditions, i.e. static pressures, were imposed in the carotid lumen and neovessels lumen respectively. Finite element method was used to simulate the mechanical conditions in the atherosclerotic plaque.

Results: Those neovessels closer to the carotid lumen undergo larger stress and stretch. With the same distance to the carotid lumen, the longer the perimeter of neovessels is, the larger stress and the deformation of the neovessels will be. Under the same conditions, the neovessels with larger curvature suffer greater stress and stretch. Neovessels surrounded by red blood cells undergo a much larger stretch.

Conclusions: Local mechanical conditions may result in the hemorrhage of neovessels and accelerate the rupture of plaque. The mechanical environments of the neovessel are related to its shape, curvature, distance to the carotid lumen and the material properties of plaque.

\section{Background}

According to the medical statistics, stroke (either ischemic or hemorrhagic) is the third leading cause of death and the primary cause of disability in the world [1,2]. In western countries, about $80 \%$ to $85 \%$ of strokes among adults are ischemic [3]. Most of the ischemic strokes are caused by the blockage in an artery that supplies blood to the brain, and hence result in a deficiency in blood flow (ischemia).

C 2015 Lu et al.; licensee BioMed Central Ltd. This is an Open Access article distributed under the terms of the Creative Commons Attribution License (http://creativecommons.org/licenses/by/4.0), which permits unrestricted use, distribution, and reproduction in any medium, provided the original work is properly cited. The Creative Commons Public Domain Dedication waiver (http:// creativecommons.org/publicdomain/zero/1.0/) applies to the data made available in this article, unless otherwise stated. 
Atherosclerotic plaque rupture is the main cause of stroke and may occur without any warning [4-7]. In the process of development and operation, atherosclerotic plaques may suddenly rupture, causing plaque debris flow and intraluminal thrombosis. Researches have shown that plaque instability is caused by cerebral infarction on the nervous system, such as a risk factor for severe damage [8]. So it is very important to judge the stability of atherosclerotic plaque for the prevention and treatment of vital stroke. Nevertheless, clinical assessment of stroke risk is still mainly based on the degree of luminal stenosis severity as measured [9]. However, more and more evidences suggest that degree of luminal stenosis alone is insufficient for identifying the critical condition [10].

Studies have demonstrated the correlation between large lipid rich necrotic core with a thin or ruptured fibrous and atherosclerotic plaque rupture [11]. Some other factors, such as plaque inflammation, fissured plaque, sex differences and intraplaque hemorrhage, are also considered [12-15]. Studies found that in the event of a plaque in patients with rupture hemorrhage caused by plaque, the detection rate of neovessels is very high $[16,17]$. Besides these factors, the mechanism of reducing plaque stability is unspecified for the neovessels in the plaque under physiological conditions. Pathological neovessel can be identified in early atherosclerosis. There is growing number of evidences suggesting that intraplaque neovessels are closely associated with intraplaque hemorrhage (IHP). But how do intraplaque neovessels promote IPH needs further investigation.

Finite element method is widely used in the biomechanical field. It can be used to predict plaque vulnerability based on peak plaque stress using human samples [18]. By using finite element method, computational models combing mechanical factors and morphologic information can be employed to implement plaque mechanical analysis, and identify additional critical mechanical factors so as to improve the current assessment criteria of plaque vulnerability based on histology and image [19-23]. Teng et al performed finite element analysis of mechanics in plaque with neovessels and showed that there are large degrees of deformation and high variation in the mechanical loading around intraplaque neovessels during the cardiac cycle [24]. Finite element analysis method can be used to quantify the critical mechanical conditions around neovessels and characterize the association between these conditions and plaque's pathological features, such as the distribution of red blood cells (RBCs) as a marker of IHP. Experimental studies have repeatedly confirmed that ischemia hypoxia is the basic cause of intraplaque angiogenesis $[25,26]$, while there is no specific law to follow about the size and shape of the neovessels.

The objective of this study is to further investigate the relationship between the critical mechanical conditions (stress and stretch) around neovessels with the morphological specificity (perimeter and curvature) and the distance to the main vessel lumen. The purpose of this paper is to evaluate the stability of plaque and provide a new way for the clinical assessment of stroke risk.

\section{Material and methods}

The present study was performed using computational structural analysis based on two carotid plaque samples which were collected with endarterectomy for histopathological examination from Department of Neurology, Beijing Tian Tan Hospital, with patient consent obtained. One of them contains lipid core while another not. In the process of staining, the lipid core occurred shedding in S2 (sample 2). The patient's blood pressures were $159 \mathrm{mmHg}$ and $140 \mathrm{mmHg}$ at systole respectively. The samples were formalin-saline 
fixed, decalcified, embedded in paraffin and stained using hematoxylin and eosin (H\&E), and then stained using Platelet endothelial cell adhesion molecule-1(PECAM-1/CD31) or Actin alpha, smooth muscle aorta ( $\alpha$-SMA). Both S1(sample1) and S2(sample 2) are decalcification. Figure 1 shows the histological slice stained using H\&E. There are a great number of neovessels (Figure 1E) in the samples (which can be seen in the slice stained by $\alpha$-SMA). All contours were manually traced by using Motic DSAssistant Lite (Motic, Inc., Amoy). Because it is a manual operation, we do not deny that there are some tiny random errors. Those contours are lumen borders and plaque components.

AutoCAD (Autodesk, Inc., USA) was applied to establish the two-dimensional models of the H\&E slices containing neovessels, fibrous cap, lipid core, fresh IPH and other components called vascular area. All components were modeled as nonlinear hyper-elastic, piecewise homogeneous and incompressible materials. Mooney-Rivlin model was used to describe the material properties of the plaque [23,27]. Materials properties are governed by the strain energy density function (Eq. 1).

$$
\mathrm{W}=\mathrm{C}_{1}\left(\mathrm{I}_{1}-3\right)+\mathrm{D}_{1} \exp \left[\mathrm{D}_{2}\left(\mathrm{I}_{1}-3\right)-1\right],
$$

where $I_{1}$ is the first strain invariant and $C_{1}, D_{1}$ and $D_{2}$ are coefficients of materials respectively. The details of these coefficients are shown in Table 1 according to the studies of Teng et al. [24,28]

The blood pressures at systole of the patients $(159 \mathrm{mmHg}$ and $140 \mathrm{mmHg}$ ) were provided by the Department of Neurology, Beijing Tian Tan Hospital. Finite element analyses of static structural mechanics of atherosclerotic plaque under physiological loading were performed using package ADINA8.8.1 (ADINA R\&D, Inc., USA). Two cases with different blood pressures of patients at systole were assigned to the main vessel lumen of carotid artery, and the pressure in each neovessel was assumed to be $10 \mathrm{mmHg}$. "This value was chosen because it approximately reflects blood pressure in
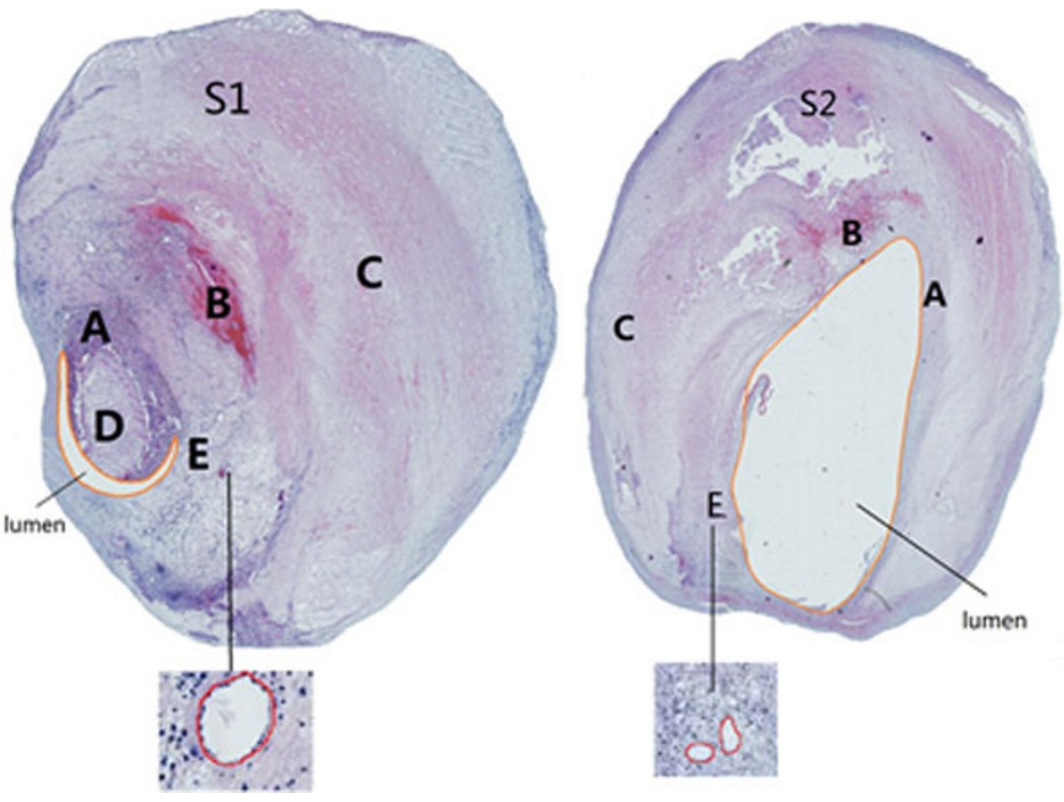

Figure 1 Two samples of microscopic slices of plaque stained using H\&E. (Left: S1; Right: S2). A: Fibrous cap; B: Fresh intraplaque hemorrhage; C: Vessel; D: Lipid core; E: Neovessels. 
Table 1 Coefficients of strain energy density function

\begin{tabular}{cccc}
\hline Components & $\mathbf{C}_{\mathbf{1}}(\mathbf{k P a})$ & $\mathbf{D}_{\mathbf{1}}(\mathbf{k P a})$ & $\mathbf{D}_{\mathbf{2}}$ \\
\hline Vessel material & 36.8 & 14.4 & 2 \\
Fibrous cap & 73.6 & 28.8 & 2.5 \\
Lipid core & 2 & 2 & 1.5 \\
Fresh IPH & 1 & 1 & 0.25 \\
\hline
\end{tabular}

the venous environment. The experimental conclusions did not change when the value was lowered to $5 \mathrm{mmHg}$ " [24]. The plaque is located inside the vessel and the carotid artery is surrounded by a lot of soft tissue, so we restricted the external displacement of plaque. Boundary conditions are illustrated in Figure 2.

In order to get more accurate results, the neovessels were divided into two groups based on its location (We checked the stress and stretch based on the grid numbers of element, as shown in Figure 2), i.e. one group in IPH area with-RBC surrounded and another group in area without-RBC surrounded. Each neovessel outline was divided into more than 10 elements, There are more than 10000 elements in both two samples. Using the MATLAB (Mathworks, Inc., USA) to calculate the shortest distance between each point and the lumen, and the minimum value was defined as the distance between neovessel and lumen. The coordinates of three adjacent points were used to calculate the curvature of the neovessels, and the maximum value of curvature was taken for further results exhibition and discussion. The association between this geometric distribution and critical mechanical condition was further analyzed.

\section{Results}

There are 167 neovessels identified in S1, 42 of them (25\%) locate in the fresh IPH area and 125 neovessels are identified in S2, 20 of them locate in the fresh IPH area. The emphases are focused on the effects of the distance to the lumen, the size and curvature of neovessels and vessel material properties, respectively.

\section{Effect of distance to lumen}

According to the distance to the lumen of the neovessels, the mechanical situations of neovessels are particularly considered. The analysis of the mechanical conditions of neovessels in plaque will be demonstrated. Two groups of neovessels with similar curvature and similar perimeter are randomly selected. The relationships between the local maximum principal stress Stress- $\mathrm{P}_{1}$ and the local maximum principal stretch Stretch- $\mathrm{P}_{1}$ with the various distances to lumen of each neovessel are shown in Figure 3.

With the increase of the distance between neovessel and lumen, the Stress- $\mathrm{P}_{1}$ and Stretch- $\mathrm{P}_{1}$ for each neovessel are decreased. The mechanical condition for intraplaque neovessels is affected by the pressure of carotid artery. Therefore, it can be deduced that for the neovessel located in the region of the same material, the closer to the lumen, the greater risk of plaque rupture. The same result can be observed from the other groups with similar curvature and perimeter, and from both samples whether located in IPH area or not.

\section{Effect of size of vessels}

The sizes of neovessels in atherosclerotic plaques are different. According to the perimeter, neovessels are divided into two groups (one group with the larger perimeter 


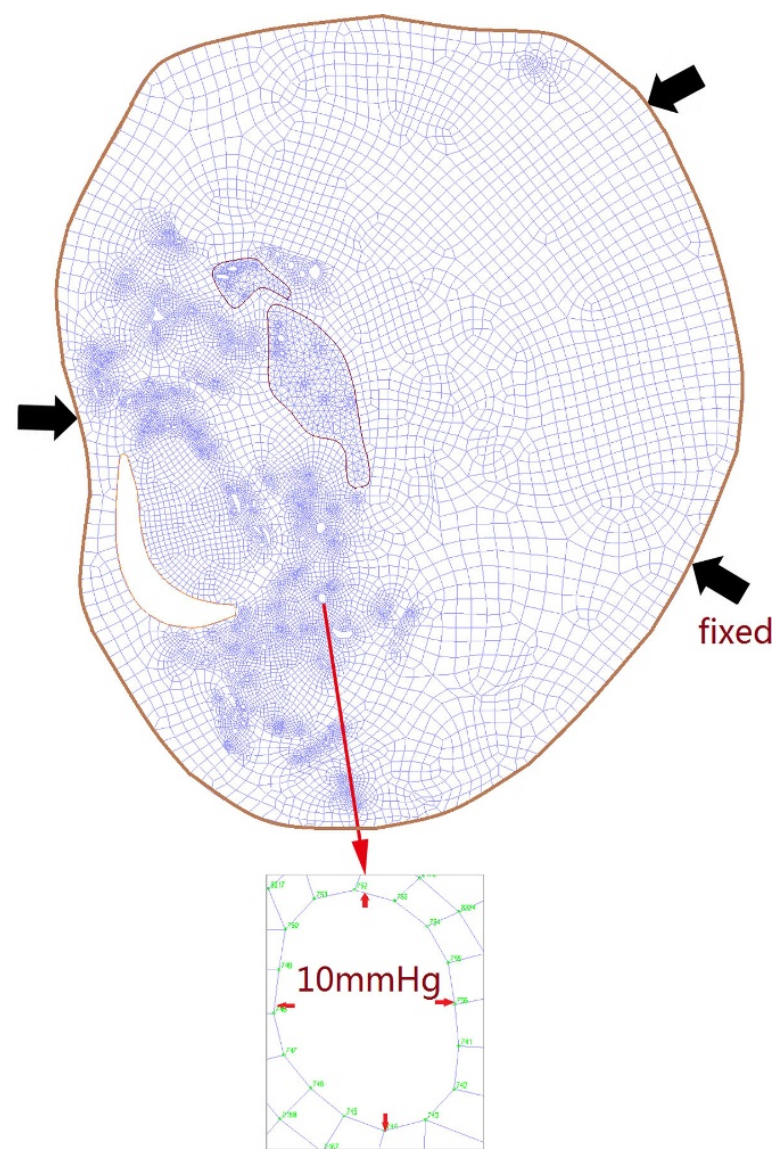

Figure 2 The 2D models for finite element analysis

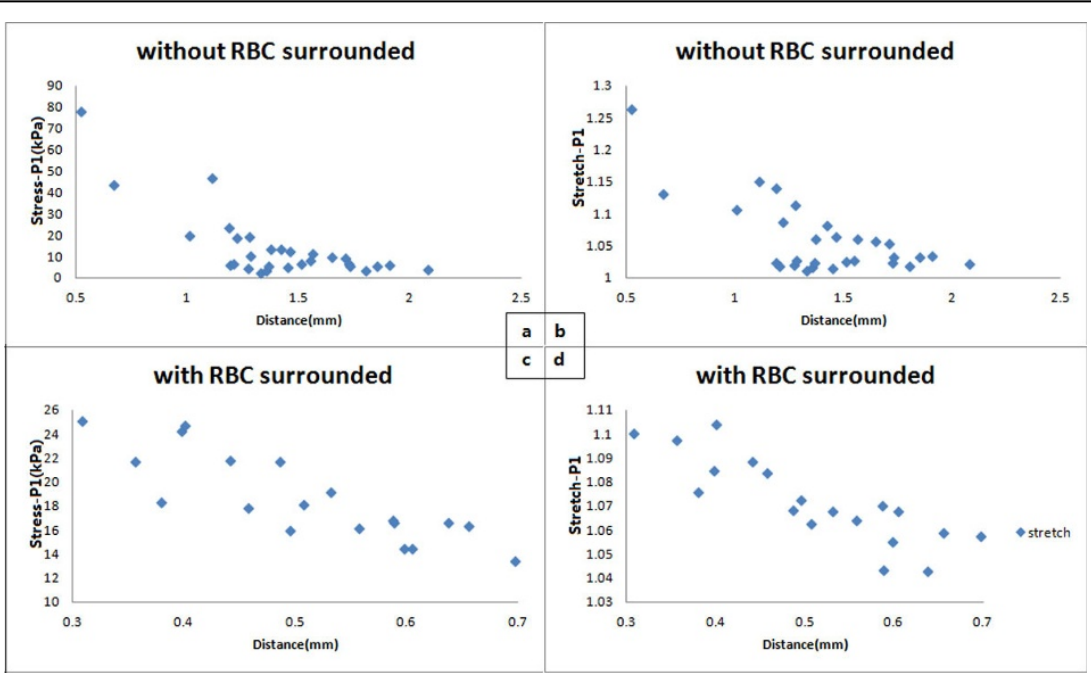

Figure 3 The relationship between critical mechanical conditions around the neovessels and their distance from the main arterial lumen. (a) local maximum principal stress (Stress-P1) at systole when neovessels without red blood cells surrounded; (b) local maximum principal stretch (Stretch-P1) at systole when neovessels without red blood cells surrounded; (c) local maximum principal stress (Stress-P1) at systole when neovessels with red blood cells surrounded; (d) local maximum principal stretch (Stretch-P1) at systole when neovessels with red blood cells surrounded. 
than the average and the other group with the smaller perimeter than the average). The relationship between the mechanical condition and the perimeter for each neovessel is evaluated by comparing the Stress-P1 and Stretch-P1 of every two neovessels with different size and similar maximum curvature in the area with equal distance to the lumen. The number of neovessels ranges from 5 to 30 in each comparison group of the same distance to lumen. Figure 4 illustrates the result of the effect of the size on its mechanical condition.

As is shown in Figure 4, in the areas with the same distance to lumen, the larger the perimeter of neovessel is, the greater stress (Figure 4a) and deformation (Figure $4 \mathrm{~b}$ ) the neovessel suffers. The same result is observed in the area with-RBC surrounded and in area without-RBC surrounded (Figure 4c, d).

\section{Effect of the curvature}

Whether the mechanical situation of the neovessels will be changed if the curvature of the neovessel is different? By using the MATLAB to calculate the curvature of each neovessel, the neovessels with the same size and the same distance to the lumen were chosen to compare the mechanical conditions between the neovessels with the maximum and the minimum curvature. The results are shown in Figure 5.

Under the same conditions, the neovessel with larger curvature suffers greater stress and stretch in the area without-RBC (Figure 5a \& 5b). The same result can also be found in the area with-RBC (Figure $5 c$ \& $5 \mathrm{~d}$ ).

\section{Effect of vessel material properties}

In the abovementioned results, the neovessels are divided into two groups base on its location in IPH area or not (i.e., with-RBC and without-RBC). The neovessels in S1 (the neovessels in IPH area in S2 are too lack to get accurate analysis result) are chosen to compare the local maximum principal stress (Stress-P1) and stretch (Stretch-P1)

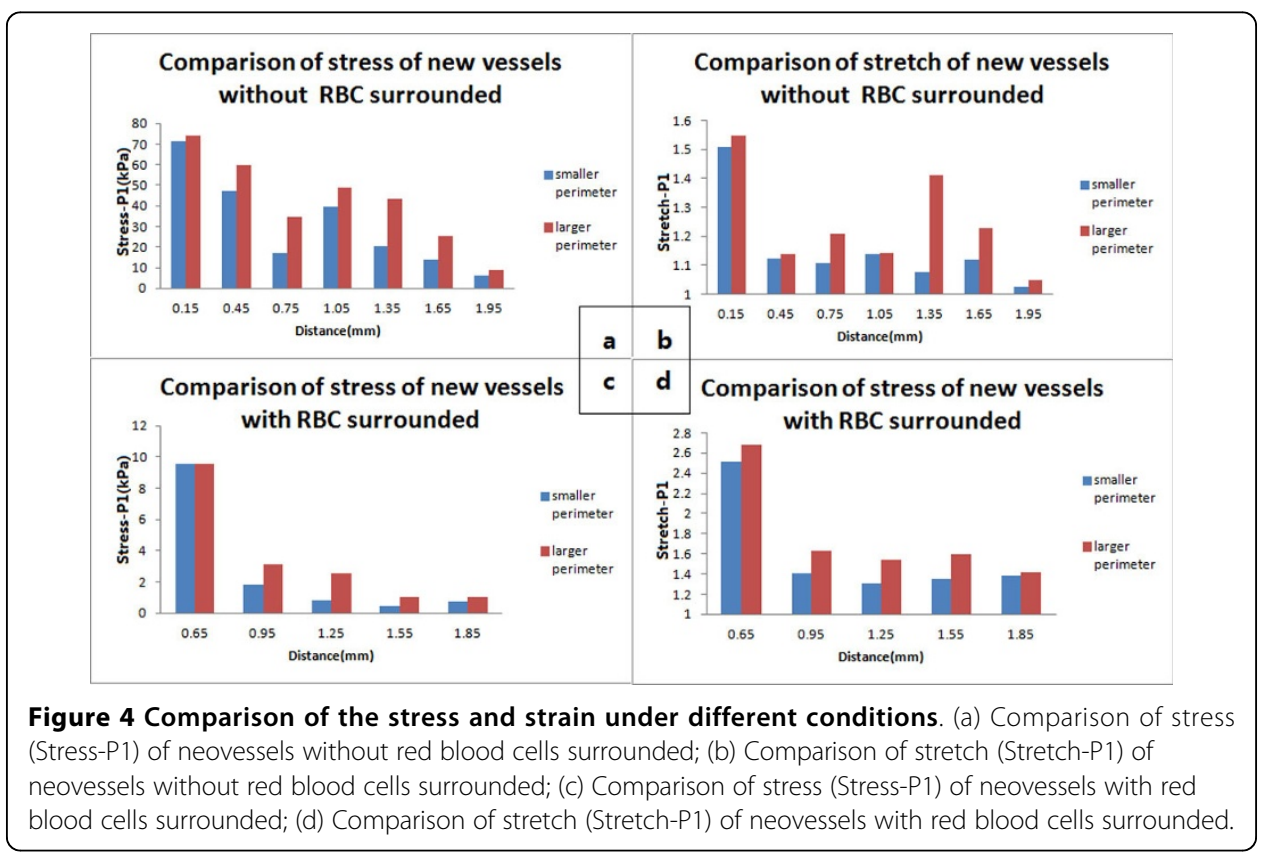




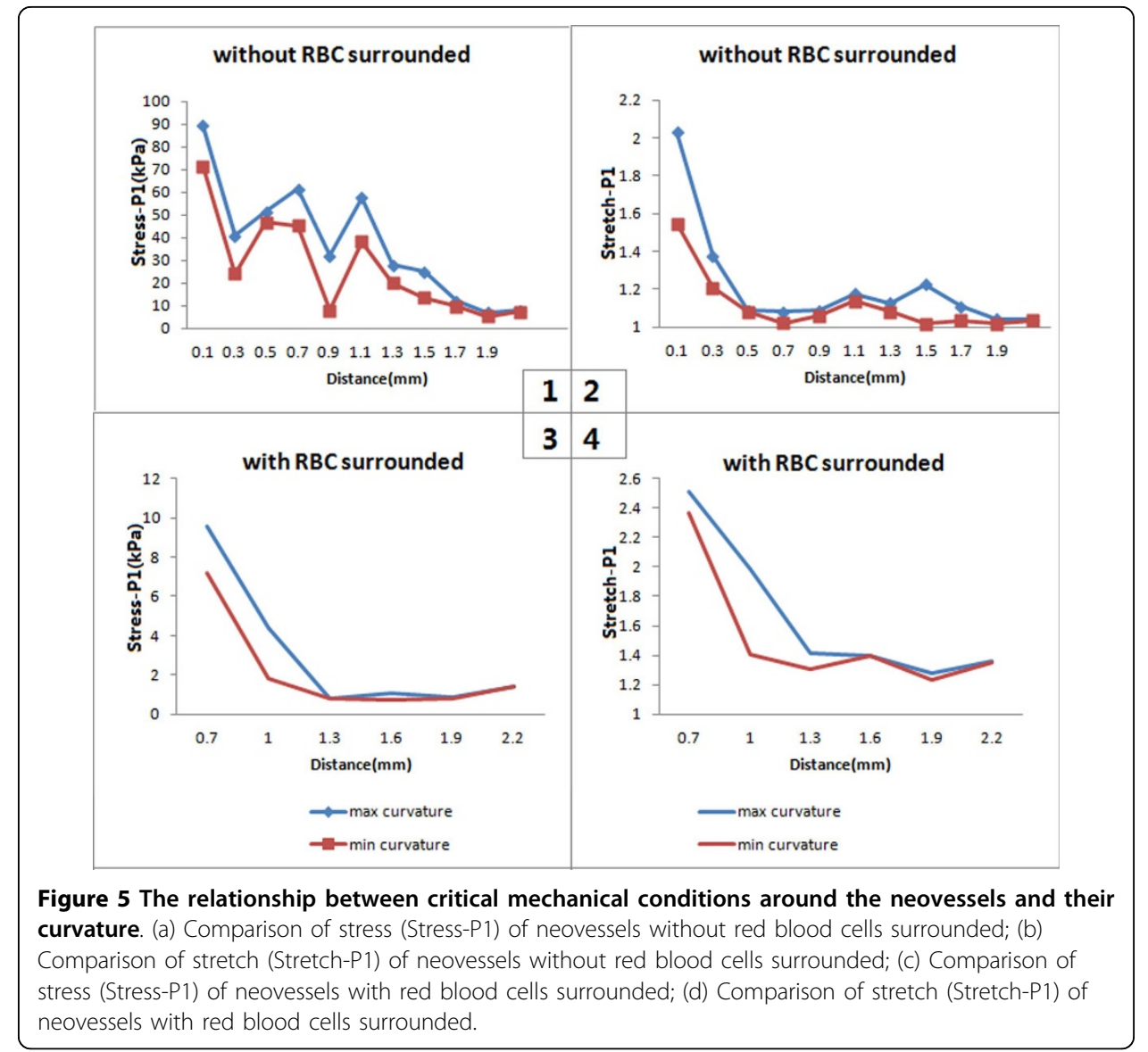

between neovessels with and without RBC surrounded. Table 2 compares the local maximum stress and stretch between neovessels with and without-RBC surrounded.

Under the pressure of carotid artery, the neovessels with-RBC surrounded undergo greater stretch than those without-RBC surrounded. The neovessels are more likely to deform when they locate in IPH area.

\section{Discussion}

Histopathological examinations have revealed the association between IPH and the presence of neovessels [29-31]. Neovascularization is the process of generating neovessels mediated primarily by progenitor and/or endothelial cells leading to tube formation, resulting in a stabilized neovascular channel [29,32]. The neovessels are born of the outer membrane of nourishing and distributed from the epicardial fat to the plaque throughout vessel wall [33]. Blood components, such as RBC, neutrophils and other proinflammatory cells, may migrate from the bloodstream into the plaque because of the incomplete development of the vascular wall about angiogenesis [24,34,35]. Under this condition, the plaque is more likely to distort, and further develop IPH. In previous studies, investigators are more willing to discuss the impacts of the plaque size and stenotic degree, the inflammatory factors and the arterial pressure, and so on [3,28,36-38]. The structure of vulnerable plaque contains the following features: (a) large lipid core; (b) high density of macrophages; (c) low density of smooth muscle cells in the cap; (d) high tissue factor content; (e) thin plaque cap in which the collagen 
Table 2 Comparison of the local maximum stress and stretch between neovessels withand without-RBC surrounded

\begin{tabular}{ccccc}
\hline Distance $(\mathbf{m m})$ & $\mathbf{0 - 0 . 6}$ & $\mathbf{0 . 6 - 1 . 2}$ & $\mathbf{1 . 2 - 1 . 8}$ & $\mathbf{1 . 8 -}$ \\
\hline Stress-P1 (with-RBC) (kPa) & {$[12.92,89.29]$} & {$[5.46,61.33]$} & {$[6.23,43.40]$} & {$[1.55,25.26]$} \\
Stress-P1 (without-RBC) (kPa) & - & {$[0.63,10.08]$} & {$[0.44,3.14]$} & {$[0.42,1.87]$} \\
Stretch-P1 (with-RBC) & {$[1.08,2.03]$} & {$[1.02,1.23]$} & {$[1.02,1.41]$} & {$[1.01,1.14]$} \\
Stretch-P1 (without-RBC) & - & {$[1.22,2.68]$} & {$[1.26,1.79]$} & {$[1.28,1.49]$} \\
\hline
\end{tabular}

structure is disorganized [39]. However, the neovessels in the plaque should not be ignored $[17,29,40]$.

The motivation of the present study is trying to explain the mechanisms about the neovessels in plaque from the biomechanical insights. As known to all, the rupture of plaque is mainly due to the stress and strain suffered, so two-dimensional models containing neovessels and other components were built according to the plaque slice. The main findings of finite element analysis are as follows.

(1) The stress and strain decrease with the increase of the distance between neovessel and lumen, which suggests that the carotid arterial pressure may play an important role in the deformation of neovessels. There is no direct association between the two groups of neovessels (i.e. one group in IPH area with-RBC surrounded and another group in area without-RBC surrounded), so we didn't discuss the comparison of stress and strain values of them. The neovessels in the plaque suffered a cyclic load with the pulsatile heartbeat. The stress and strain/stretch changes periodically. The neovessels may fatigue under cyclic loading, and those close to lumen are more dangerous.

(2) The neovessel with larger perimeter suffers much greater deformation, which indicates that the size is a significant factor for the fatigue of neovessels. The neovessels features high permeability and poor stability. Thus, the longer perimeter they have, the higher risk of rupture they face. Because the mechanical environments of the neovessels are related to integrated conditions including its shape, curvature, distance to the carotid lumen and the material properties of plaque, etc. We intended to show the result to express the influence of a single factor condition; however those figures in Figure 4 can not isolate the single factor while showing the total values of stress and stretch. We defined the neovessel with equal distance to lumen (per $0.3 \mathrm{~mm}$ ) and similar curvature values (difference within ten times) for a group to investigate the effect of size on neovessels. We believe that the contradiction is due to the overall impact of the whole integrated factors.

(3) Under the same conditions, the neovessel with larger curvature suffers greater stress and stretch. The great curvature of neovessel with "sharp" edge generates stress concentration which is more easily to induce hemorrhage rupture.

(4) Those neovessels with-RBC surrounded undergo greater Stretch-P1 than those without-RBC surrounded. It indicates that the location of neovessels in plaque is also a significant factor for the deformation of neovessels. The hostile mechanical environment around neovessels may be concerned with the divulging of RBCs, which can be found around those neovessels suffered a large deformation. However, there is no significant difference of Stress-P1 between the groups with-RBC and without-RBC.

Although the angiogenesis can be identified as an effective means of increasing myocardial perfusion, the relationship between neovessels and plaque instability cannot be 
ignored yet. Our study demonstrates that neovessels in plaque rupture and hemorrhage may promote the plaque rupture, and increase the risk of stroke.

Some limitations in this study must be mentioned. First, only two samples with total 292 neovessels were modeled, which may not be enough for the strict verification to support our hypothesis. Some factors, such as the density of neovessels ought to be considered. Second, this study was a two-dimensional simulation, and the effect of the blood flow was not taken into account. The flow in the lumen and the week flow in the neovessels need to be considered for further hemodynamic calculation. In the process of staining, the lipid core occurred shedding in S2, so we ignored it when performing the numerical simulation analysis. But the results are within very small discrepancy and still support our forecast.

\section{Conclusions}

Our hypothesis is that the mechanical situations of intraplaque neovessel are largely due to the complexity of biomechanical interactions. We suggest that there are large degrees of stress and deformation by the mechanical loading around the neovessels in plaque. By using the numerical method to analyze the mechanical conditions of neovessels in the plaque, we found that local mechanical conditions contribute to the neovessel damage and further IPH formation. Results show that those neovessels closer to the carotid lumen undergo larger stress and stretch. With the same distance to the carotid lumen, the longer is the perimeter of neovessels, the larger stress and the deformation are generated on the neovessels. Neovessels surrounded by red blood cells undergo a much larger stretch. Local mechanical conditions may result in the hemorrhage of neovessels and accelerate the rupture of plaque. The mechanical environments of the neovessels are related to its shape, curvature, distance to the carotid lumen and the material properties of plaque. The finding of this paper may be applied to evaluate the stability of plaque and provide a new way for the clinical assessment of stroke risk.

\section{Ethics statement}

All human data used in this study were obtained from consenting patients of the Neural Department of Internal Medicine in Beijing Tian Tan Hospital, and was approved by the hospital's Ethical Research Committee.

Competing interests

Other than the grants listed in the acknowledgment section, the authors declare that they have no other competing interests.

\section{Authors' contributions}

All authors 1) have made substantial contributions to conception and design, or acquisition of data, or analysis and interpretation of data; 2) have been involved in drafting the manuscript or revising it critically for important intellectual content; and 3) have given final approval of the version to be published. Each author has participated sufficiently in the work to take public responsibility for appropriate portions of the content.

\section{Acknowledgements}

These researches are supported by National Natural Science Foundation of China (81171107, 81341117), Beijing Science Foundation (KZ201210005006) and Specialized Research Fund for the Doctoral Program of Higher Education (20111103110012)

\section{Declarations}

Publication of this article was paid with funding from National Natural Science Foundation of China (81171107). This article has been published as part of BioMedical Engineering OnLine Volume 14 Supplement 1, 2015 :

Cardiovascular Disease and Vulnerable Plaque Biomechanics. The full contents of the supplement are available online at http://www.biomedical-engineering-online.com/supplements/14/S1 


\section{Authors' details}

${ }^{1}$ College of Life Science and Bioengineering, Beijing University of Technology, Beijing, 100124, China. ${ }^{2}$ Neural Department of Internal Medicine, Beijing Tian Tan Hospital, Capital Medical University, Beijing, 100050, China.

Published: 9 January 2015

\section{References}

1. Mathers C, Fat DM, Boerma JT: The global burden of disease: 2004 update. World Health Organization 2008

2. Donnan GA, Fisher M, Macleod M, Davis SM: Stroke. The Lancet 2008, 371:1612-1623.

3. Hocker S, Morales-Vidal S, Schneck MJ: Management of Arterial Blood Pressure in Acute Ischemic and Hemorrhagic Stroke. Neurologic ClinicsAdvances in Neurologic Therapy 2010, 28:863-886.

4. Naghavi M, Libby P, Falk E, Casscells SW, Litovsky S, Rumberger J, Badimon JJ, Stefanadis C, Moreno PR, Pasterkamp G: From vulnerable plaque to vulnerable patient: a call for new definitions and risk assessment strategies: Part II. Circulation (Baltimore) 2003, 108:1772-1778.

5. Naghavi M, Libby P, Falk E, Casscells SW, Litovsky S, Rumberger J, Badimon JJ, Moreno P, Pasterkamp G: From Vulnerable Plaque to Vulnerable Patient A Call for New Definitions and Risk Assessment Strategies: Part I. Circulation 2003, 108:1664-1672.

6. Fuster V: The vulnerable atherosclerotic plaque: understanding, identification, and modification. Cardiovascular Drugs and Therapy 1999, 13:363.

7. Huang X, Teng Z, Canton G, Ferguson M, Yuan C, Tang D: Intraplaque hemorrhage is associated with higher structural stresses in human atherosclerotic plaques: an in vivo MRI-based $3 \mathrm{~d}$ fluid-structure interaction study. Biomedical engineering online 2010, 9:86.

8. Liu F, Yao Y, Tian Y, Zhang W, Hao X, Ma H: Pathologic analysis of the relationship between cerebral stroke and atheromatous lesions of extracranial carotid artery. Chinese Journal of Neurology 1998, 5 .

9. Inzitari D, Eliasziw M, Gates P, Sharpe BL, Chan RK, Meldrum HE, Barnett HJ: The causes and risk of stroke in patients with asymptomatic internal-carotid-artery stenosis. New England Journal of Medicine 2000, 342:1693-1701.

10. Pasterkamp G, Smits PC: Remodelling of coronary arteries. Journal of cardiovascular risk 2002, 9:229-235.

11. Zhao X, Dong L, Hatsukami T, Phan BA, Chu B, Moore A, Lane T, Neradilek MB, Polissar N, Monick D: MR Imaging of Carotid Plaque Composition During Lipid-Lowering TherapyA Prospective Assessment of Effect and Time Course. JACC: Cardiovascular Imaging 2011, 4:977-986.

12. Ota H, Reeves MJ, Zhu DC, Majid A, Collar A, Yuan C, DeMarco JK: Sex differences of high-risk carotid atherosclerotic plaque with less than $50 \%$ stenosis in asymptomatic patients: an in vivo $3 \mathrm{~T} \mathrm{MRI} \mathrm{study.} \mathrm{American} \mathrm{Journal} \mathrm{of}$ Neuroradiology 2013, 34:1049-1055.

13. Kerwin WS, Oikawa M, Yuan C, Jarvik GP, Hatsukami TS: MR imaging of adventitial vasa vasorum in carotid atherosclerosis. Magnetic Resonance in Medicine 2008, 59:507-514.

14. Kolodgie FD, Gold HK, Burke AP, Fowler DR, Kruth HS, Weber DK, Farb A, Guerrero LJ, Hayase M, Kutys R: Intraplaque hemorrhage and progression of coronary atheroma. New England Journal of Medicine 2003, 349:2316-2325.

15. Virmani R, Kolodgie FD, Burke AP, Farb A, Schwartz SM: Lessons from sudden coronary death a comprehensive morphological classification scheme for atherosclerotic lesions. Arteriosclerosis, thrombosis, and vascular biology 2000, 20:1262-1275.

16. Xiong L, Deng Y, Zhu Y, Liu Y, Bi X: Correlation of carotid plaque neovascularization detected by using contrastenhanced US with clinical symptoms. Radiology 2009, 251:583.

17. Chen F, Eriksson P, Kimura T, Herzfeld I, Valen G: Apoptosis and angiogenesis are induced in the unstable coronary atherosclerotic plaque. Coronary artery disease 2005, 16:191-197.

18. Karimi A, Navidbakhsh M, Faghihi S, Shojaei A, Hassani K: A finite element investigation on plaque vulnerability in realistic healthy and atherosclerotic human coronary arteries. Proceedings of the Institution of Mechanical Engineers Part H Journal of Engineering in Medicine 2013, 227:148-161.

19. Kaazempur-Mofrad MR, Isasi AG, Younis HF, Chan RC, Hinton DP, Sukhova G, LaMuraglia GM, Lee RT, Kamm RD: Characterization of the atherosclerotic carotid bifurcation using MRI, finite element modeling, and histology. Annals of biomedical engineering 2004, 32:932-946.

20. Sadat U, Teng Z, Young VE, Zhu C, Tang TY, Graves MJ, Gillard JH: Impact of plaque haemorrhage and its age on structural stresses in atherosclerotic plaques of patients with carotid artery disease: an MR imaging-based finite element simulation study. The International Journal of Cardiovascular Imaging 2011, 27:397-402.

21. Tang D, Teng Z, Canton G, Hatsukami TS, Dong L, Huang X, Yuan C: Local critical stress correlates better than global maximum stress with plaque morphological features linked to atherosclerotic plaque vulnerability: an in vivo multi-patient study. Biomed Eng Online 2009, 8:15.

22. Tang D, Teng Z, Canton G, Yang C, Ferguson M, Huang X, Zheng J, Woodard PK, Yuan C: Sites of Rupture in Human Atherosclerotic Carotid Plaques Are Associated With High Structural Stresses An In Vivo MRI-Based 3D FluidStructure Interaction Study. Stroke 2009, 40:3258-3263.

23. Tang D, Yang C, Zheng J, Woodard PK, Sicard GA, Saffitz JE, Yuan C: 3D MRI-based multicomponent FSI models for atherosclerotic plaques. Annals of biomedical engineering 2004, 32:947-960.

24. Teng Z, He J, Degnan AJ, Chen S, Sadat U, Bahaei NS, Rudd JHF, Gillard JH: Critical mechanical conditions around neovessels in carotid atherosclerotic plaque may promote intraplaque hemorrhage. Atherosclerosis 2012, 223:321-326.

25. Galili O, Sattler KJ, Herrmann J, Woodrum J, Olson M, Lerman LO, Lerman A: Experimental hypercholesterolemia differentially affects adventitial vasa vasorum and vessel structure of the left internal thoracic and coronary arteries. The Journal of thoracic and cardiovascular surgery 2005, 129:767-772.

26. Williams JK, Armstrong ML, Heistad DD: Vasa vasorum in atherosclerotic coronary arteries: responses to vasoactive stimuli and regression of atherosclerosis. Circulation research 1988, 62:515-523.

27. Bathe KJ: Theory and Modeling Guide. Watertown, MA: ADINA R \& D.: Inc. 2002. 
28. Little WC, Applegate RJ: Role of plaque size and degree of stenosis in acute myocardial infarction. Cardiology Clinics $1996,14: 221-228$

29. Moreno PR, Purushothaman K, Sirol M, Levy AP, Fuster V: Neovascularization in human atherosclerosis. Circulation 2006, 113:2245-2252.

30. Moreno PR, Purushothaman KR, Fuster V, Echeverri D, Truszczynska H, Sharma SK, Badimon JJ, O Connor WN: Plaque neovascularization is increased in ruptured atherosclerotic lesions of human aorta implications for plaque vulnerability. Circulation 2004, 110:2032-2038.

31. Milei J, Parodi JC, Alonso GF, Barone A, Grana D, Matturri L: Carotid rupture and intraplaque hemorrhage: Immunophenotype and role of cells involved. American Heart Journal 1998, 136:1096-1105.

32. Risau W: Mechanisms of angiogenesis. Nature 1997, 386:671-674.

33. Barger AC, Beeuwkes Rd R, Lainey LL, Silverman KJ: Hypothesis: vasa vasorum and neovascularization of human coronary arteries. A possible role in the pathophysiology of atherosclerosis. The New England journal of medicine 1984, 310:175.

34. Leclercq A, Houard X, Philippe M, Ollivier V, Sebbag U, Meilhac O, Michel J: Involvement of intraplaque hemorrhage in atherothrombosis evolution via neutrophil protease enrichment. Journal of leukocyte biology 2007, 82:1420-1429.

35. Virmani R, Kolodgie FD, Burke AP, Finn AV, Gold HK, Tulenko TN, Wrenn SP, Narula J: Atherosclerotic plaque progression and vulnerability to rupture angiogenesis as a source of intraplaque hemorrhage. Arteriosclerosis, thrombosis, and vascular biology 2005, 25:2054-2061.

36. de Labriolle A, Mohty D, Pacouret G, Giraudeau B, Fichet J, Fremont B, Fauchier L, Charbonnier B, Arbeille P: Comparison of Degree of Stenosis and Plaque Volume for the Assessment of Carotid Atherosclerosis Using 2-D Ultrasound. Ultrasound in Medicine \& Biology 2009, 35:1436-1442.

37. Zaman AG, Helft G, Worthley SG, Badimon Jj: The role of plaque rupture and thrombosis in coronary artery disease. Atherosclerosis 2000, 149:251-266.

38. Rothwell PM, Salinas R, Ferrando LA, Slattery J, Warlow CP: Does the angiographic appearance of a carotid stenosis predict the risk of stroke independently of the degree of stenosis? Clinical Radiology 1995, 50:830-833.

39. Davies MJ: The pathophysiology of acute coronary syndromes. Heart 2000, 83:361-366.

40. Calcagno C, Cornily J, Hyafil F, Rudd JH, Briley-Saebo KC, Mani V, Goldschlager G, Machac J, Fuster V, Fayad ZA: Detection of neovessels in atherosclerotic plaques of rabbits using dynamic contrast enhanced MRI and 18F-FDG PET. Arteriosclerosis, thrombosis, and vascular biology 2008, 28:1311-1317.

doi:10.1186/1475-925X-14-S1-S3

Cite this article as: Lu et al:: Finite element analysis of mechanics of neovessels with intraplaque hemorrhage in carotid atherosclerosis. BioMedical Engineering OnLine 2015 14(Suppl 1):S3.

\section{Submit your next manuscript to BioMed Central and take full advantage of:}

- Convenient online submission

- Thorough peer review

- No space constraints or color figure charges

- Immediate publication on acceptance

- Inclusion in PubMed, CAS, Scopus and Google Scholar

- Research which is freely available for redistribution 